\title{
Nuevas perspectivas en la gestión de las administraciones públicas: una visión comparada de la contratación pública electrónica
}

\author{
Valdemir APARECIDO PIRES \\ Grupo de Pesquisa «Controle Social do Gasto Público» UNESP pires.valdemir@gmail.com \\ Carmen PinEDA NeBOT \\ Grupo de Pesquisa «Controle Social do Gasto Público» UNESP carmenpinedanebot@hotmail.com \\ Jordi ROMEU GRANADOS \\ Grupo de Pesquisa «Controle Social do Gasto Público» UNESP jromeugranados@gmail.com \\ Gregorio JUÁREZ RODRÍGUEZ \\ Grupo de Pesquisa «Controle Social do Gasto Público» UNESP gregorijuarez13@gmail.com
}

Recibido: $15 / 10 / 2014$

Aceptado: 04/12/2014

\begin{abstract}
Resumen
La progresiva incorporación de medios electrónicos en la actividad pública no solo supone una «modernización», sino que está implicando una profunda transformación del modelo de gestión, abriendo nuevas posibilidades a la participación de los actores y al control. En el presente trabajo se analiza, de un modo comparado, dos de los modelos existentes en materia de contratación pública electrónica, pertenecientes a ámbitos político-territoriales diferenciados y con trayectorias también diferentes, como son el español y el brasileño. Se muestran los procesos evolutivos seguidos en ambos casos, los problemas y dificultades a los que se enfrentan, las soluciones planteadas y la situación en la que se encuentran en estos momentos.
\end{abstract}

Palabras clave: gestión pública, innovación, contratación pública, e-Contratación.

New perspectives on public administrations management: a comparative view of electronic public procurement

\begin{abstract}
The incorporation of technology to public activity brings us not only a «modernization», it is creating deep transformations in the management model, opening news opportunities to stakeholders' participation and to the control. In this paper we analyze, in a comparative way, two of the current models of electronic public procurement, which come from political-territorial different areas with different development too: Spain and Brazil. We show the evolution in both cases, the problems and difficulties they have to deal with, the solutions given, and the conditions they are living nowadays.
\end{abstract}

Key words: public management, innovation, public procurement, e-Procurement.

\section{Introducción}

La progresiva incorporación de medios electrónicos en la actividad pública no solo supone una «modernización» en los instrumentos de que se sirve el poder para desarrollar sus funciones, sino que está implicando una profunda transformación del mode- 
lo de gestión, abriendo nuevas posibilidades a la participación de los actores y al control, lo que necesariamente redunda en beneficio de la sociedad (Romeu et al., 2014). La transformación se muestra necesaria en un momento en que existe una fuerte crisis global, tanto económica como política, que exige la adaptación de las instituciones y políticas públicas mediante la puesta en práctica de los principios e instrumentos de gobernanza $^{1}$. De este modo, la innovación, en el marco de la gobernanza ${ }^{2}$, conlleva modificaciones estructurales y aúna grandes esfuerzos de transparencia, coordinación, participación ciudadana y feedback en la rendición de cuentas, implica una reorientación hacia la asociación o la cooperación interorganizacional en torno a objetivos comunes e introduce cambios en los procesos de decisión, en la distribución de poder y recursos (financieros, cognitivos, etc.), en los criterios y normas sobre el funcionamiento de las redes organizacionales, y en sus políticas o estrategias de actuación (Zapico, 2012:9), siendo la actividad contractual pública, conformada por una pluralidad de actores e intereses públicos y privados en juego (económicos, empresariales, políticos, administrativos, sociales, etc.), uno de los más significativos exponentes de dicho modelo. La complejidad y las dificultades de la innovación transformacional no provienen tanto de la dificultad técnica del cambio sino de la diversidad de valores e intereses y los efectos del cambio en los equilibrios de poder y colaboración.

Así pues, la utilización de los medios electrónicos en el ámbito de las contrataciones públicas supone una mejora en algunos de los principios tradicionalmente asociados a la misma, como pueden ser el de transparencia ${ }^{3}$ o el de igualdad en las condiciones de acceso a los procedimientos de licitación. Lo que implica, a su vez, una optimización del gasto público ${ }^{4}$, pues una mayor difusión de las licitaciones permite el aumento de la concurrencia, la mejora en las ofertas realizadas, y un adecuado segui-

1 Aunque este modelo es bien conocido, sobre todo en el mundo de las ciencias políticas y de la administración pública, la implementación del mismo se ha desarrollado con cierta timidez.

2 Esta puede definirse como un modelo de decisión orientado a satisfacer las preferencias sociales definidas entre el Gobierno y la sociedad y realizadas con acciones, recursos, herramientas y formas de organización consensuadas (Aguilar y Bustelo, 2010; Villoria, 2010). En este modelo, el Gobierno es un actor más, con un nivel relativo de influencia, que actúa a través de redes de colaboración, junto con otros niveles de Gobierno y actores económicos y sociales. Su papel no es tanto diseñar y ejecutar políticas públicas, rindiendo cuentas de los servicios prestados de forma supuestamente independiente y totalmente responsable, sino contribuir a la creación y fortalecimiento de redes, promover procesos de participación, acuerdos de colaboración, coordinación y resolución de conflictos, y compartir la responsabilidad con otros niveles de Gobierno y la sociedad por el funcionamiento y la mejora de las políticas y las instituciones públicas (Zapico, 2012:8).

${ }^{3}$ La e-Contratación se manifiesta como un instrumento que per se facilita la transparencia (Joongi, 2006; González Alonso, 2009; Vaidya, 2009; Gardenal, 2010; Moreno Molina, 2011; Elezi y Harizaj, 2012; Neupane et al., 2012a, 2012b; Alaweti et al., 2013; Valadares Tabares, 2013; Caño, 2013; Pintos, 2014; Romeu et al., 2014a, 2014b), al promover la apertura de datos (Álvarez et al., 2011) y la divulgación de los mismos.

${ }^{4}$ Una de las partidas más importantes del gasto público es la contratación pública (alrededor del $20 \%$ del PIB), y con tendencia a aumentar. Pero su gestión arrastra aún un excesivo sesgo de un proceso de derecho administrativo y le falta, a veces de forma notoria, el sesgo económico y político, y valores como la transparencia (qué se compra) y rendición de cuentas (qué resultados se han obtenido), que todas las sociedades modernas exigen a las compras públicas (Caño, 2013). 
miento por parte de la ciudadanía de las políticas desarrolladas al respecto. Los casos de corrupción y falta de ética en las administraciones, muy relacionados con la contratación, originan -como señalan Ramírez-Alujas y Villoria (2012)- una profunda desconfianza institucional, (Pharr y Putnam, 2000; Anderson y Tverdova, 2003; Bowler y Karp, 2004) además de costes inasumibles (Ades y Di Tella, 1997; Della Porta y Vannucci, 1997; Dreher y Herzfeld, 2005; Hodgson y Jiang, 2007), entre otros efectos negativos. Las administraciones que gestionan con niveles elevados de corrupción bloquean el desarrollo del país. La corrupción impide el desarrollo porque impide gobernar eficaz y eficientemente, es por ello el mayor obstáculo al avance económico y social (World Bank, 2006) y sus efectos sobre los fundamentos sociales de las comunidades -como la confianza intersubjetiva, la confianza en las instituciones, la cultura de la legalidad- son muy negativos (Villoria y Jiménez, 2012). Pero la innovación es un concepto complejo, no quedando claro en numerosa literatura si se trata de un producto o de un proceso, una idea tomada de otros o un descubrimiento. Lo que abre la puerta a una pluralidad de enfoques y definiciones (Osborne y Brown 2005, Ramírez-Alujas 2012). Pudiendo distinguirse entre enfoques instrumentales y sustantivos. Los primeros se refieren a la innovación como un conjunto de ideas que permiten mejorar la eficiencia de determinadas actuaciones. Desde esta óptica, no se modifica lo que hacemos sino la manera de hacerlo. Los segundos, en cambio, consideran que la innovación implica una forma sustancialmente diferente de entender, abordar y transformar la realidad, no se trata solo de incidir en los procesos sino de replantear la propia naturaleza de los problemas (Brugué, Blanco y Boada, 2013).

En este análisis optamos por un enfoque sustantivo que interpreta la innovación como la capacidad de construir nuevas respuestas frente a los problemas complejos que deben abordar las políticas públicas. Por lo tanto, no se relaciona solo con nuevas técnicas sino con nuevos conocimientos. Aunque el conocimiento no se genera simplemente por acumulación de información sino que requiere de la experiencia, los juicios y las valoraciones de una amplia red de actores. Es un proceso colectivo, en el que las redes son clave para la producción (Agranoff y McGuire, 2003), es una reflexión compartida y democrática donde el nuevo conocimiento se genera a partir del intercambio (Brugué, Blanco y Boada, 2013).

Pero a pesar de las dificultades que plantea el concepto de innovación aplicado al sector público, es central en el nuevo siglo y un eslabón para fortalecer la democracia. Desde esta perspectiva, la innovación podría alinear mejor las actividades del gobierno y el sector público con las necesidades de los ciudadanos, y ayudar a resolver las fallas ( $\tan$ frecuentes) en el diseño e implementación de las políticas públicas (Moran, 2004).

En el presente trabajo analizamos uno de los ámbitos materiales de innovación de la administración pública: la contratación pública electrónica; realizando un análisis comparado de dos de los modos de adopción de la misma, pertenecientes a dos ámbitos político-territoriales diferenciados y con trayectorias también diferentes: el modelo brasileño y el modelo español. Mostramos los procesos evolutivos seguidos en ambos casos, los problemas y dificultades a los que se enfrentan, las soluciones planteadas y la situación en la que se encuentran en estos momentos. 


\section{Metodología}

El método escogido ha sido el estudio de caso comparativo, al considerar que esta técnica es adecuada en situaciones en las que el objetivo del investigador es comprender cómo las condiciones de causa o las variables de acción producen, en ciertas situaciones, efectos sobre las variables de resultado (Van Evera, 1997).

Si esas condiciones son adecuadamente estudiadas, mapeadas y comprendidas, se genera información que puede ser interpretada a partir de los mecanismos de relación teórica. En esa vertiente, esos mecanismos son tan importantes como la capacidad de generalización en sí, principalmente para las ciencias sociales aplicadas, en las que causa y efecto no son, por regla general, un mecanismo probablemente descifrable. Este estudio es comparado desde la perspectiva de la Administración Pública Comparada, que tiene el propósito de, a partir de diferentes experiencias, mejorar el desempeño de procesos o sistemas administrativos con la incorporación de buenas prácticas y el establecimiento de limitaciones.

En esa dirección, Hedstrom (2005) admite que la base de la comprensión de las explicaciones por mecanismos reside en la especificación de los mismos o de sus conexiones y de relaciones causales capaces de describir de forma más inteligible cómo un fenómeno específico o determinados resultados fueron generados a partir de dicha realidad contextual.

Por consiguiente, como nos muestra Rezende (2011), a partir de estudios de caso, los investigadores pueden utilizar las estrategias que les permitan observar de qué modo las condiciones iniciales se articulan para producir determinadas variaciones en la variable de resultado. Es recomendable acudir a este tipo de análisis cuando los investigadores se orientan a comparar explicaciones que no pueden ser consideradas con mayor profundidad en las investigaciones con grandes muestras.

Sin embargo, parece legítimo proponer que a partir de los resultados de Brasil y España sea posible, como mínimo, describir el estado de la contratación electrónica, así como señalar los problemas y dificultades a las que se enfrentan y comprender las soluciones planteadas para que se alcance el fin deseado, a partir de conclusiones válidas, siendo esa la propuesta metodológica del trabajo.

\section{Brasil}

\section{a) Antecedentes}

En Brasil, la licitación, como requisito para la realización de compras y contrataciones públicas, solo se reguló en una norma específica a partir de 1986, con el Decreto 2.300/86, conocido como Estatuto Jurídico de las Licitaciones y Contratos Administrativos. Anteriormente, este proceso se regía por normas que lo incluían junto con otros temas de la administración pública. Así, el Decreto 2.926, de 1862, que era una regulación para adjudicaciones de los servicios del Ministerio de Agricultura, Comercio y Obras Públicas; o el Decreto 4.536, de 1922, que trataba de la Contabilidad Pública (y que mencionaba la exigencia de «concurrencia» para la realización de las com- 
pras); la Ley 4.401, de 1964, que adopta por primera vez el término «licitación»; y, finalmente, el Decreto-ley n. 200, de 1967, que contiene un título denominado «De las normas relativas a las licitaciones para compras, obras, servicios y enajenaciones».

La legislación brasileña en vigor para licitaciones y contratos públicos abarca un conjunto de normas (leyes, decretos, órdenes) cuyo pilar fundamental es la ley 8.666, de 1993, enmendada muy pronto por la ley 8.883 , de 1994. En ella se crearon las modalidades de licitación que se utilizan actualmente: concurrencia, concurrencia internacional, concurso, invitación y fijación de precio; también fueron previstas situaciones para dispensa o inexigibilidad de licitaciones y las subastas para la enajenación de bienes pertenecientes a los gobiernos. Desde 1998, por la ley 9.648, estas modalidades se escogen en función de los valores financieros incluidos, de modo que las cantidades mayores exigen procedimientos más complejos, que se reducen a medida que se trata de volúmenes menores de recursos.

Recientemente fue creada y regularizada la subasta a la baja (o subasta a la inversa), admitida solamente para la adquisición de bienes o servicios comunes, o sea, «aquellos cuyos niveles de rendimiento y calidad puedan ser objetivamente definidos por el pliego de condiciones, por medio de especificaciones habituales en el mercado», como establece el párrafo único del artículo $1^{\circ}$. de la ley 10.520 , de 2002 , que es el marco legal para este procedimiento. La subasta a la baja puede ser presencial o electrónica. Esta última es la que en Brasil normalmente se considera como contratación pública electrónica (o public e-Procurement, o e-compras públicas).

La subasta a la baja se utilizó por primera vez en 1998, por la Agencia Nacional de Telecomunicaciones (ANATEL), creada en 1997, después de la privatización de los servicios de telefonía. La misma ANATEL introdujo la modalidad electrónica en 2000, año en el que el gobierno federal adoptó la subasta a la baja presencial por medio del Decreto-Ley 2.026, incluyendo después la modalidad electrónica en sus procedimientos (decreto 3.697, de diciembre de 2001). Funciona como una típica subasta a la inversa, en la cual se presentan ofertas por debajo del valor de referencia inicial, con el objetivo de simplificar el proceso de licitación, eliminar barreras a la participación de ofertantes y obtener mejores precios. La mencionada ley 10.520/2002 lo extendió a los gobiernos de todos los niveles de la federación (Unión, Estados y Municipios).

Convergiendo, como factores que impulsan la adhesión del gobierno a los procedimientos de public e-Procurement, el avance de las tecnologías de la información y comunicación (TIC) característico de las últimas décadas; el movimiento reformista del Estado de los años 80-90; y el generalizado descontento (dentro y fuera del gobierno) con la morosidad e ineficiencia de los procesos de licitación en el país, regidos por la ley $8.666 / 83$.

\section{b) La experiencia de la subasta a la baja electrónica en el gobierno federal}

El gobierno federal adoptó rápidamente la subasta a la baja y la subasta a la baja electrónica, en busca de agilidad, economía, mayor apoyo de potenciales proveedores, transparencia y combate a la corrupción (muy común en las licitaciones, no solo en Brasil, sino en todo el mundo). Para ello implementó una política permanente de inno- 
vación en este campo, cuyo núcleo central es el portal de compras del gobierno federal en internet, el Comprasnet ${ }^{5}$ (http://www.comprasnet.gov.br/, ver Figura 1). Este se creó por el Decreto $\mathrm{n}^{\circ}$ 1.094, de 1994, y en la actualidad es administrado por el Ministerio de Planificación, Presupuesto y Gestión, a partir del Sistema Integrado de Administración de Servicios Generales (SIASG). El propio portal Comprasnet se define a sí mismo como «un conjunto informatizado de herramientas para hacer internamente operativo el funcionamiento sistémico de las actividades inherentes al Sistema de Servicios Generales - SISG, que son: la gestión de materiales, edificaciones públicas, vehículos oficiales, comunicaciones administrativas, licitaciones y contratos, de los cuales el Ministerio de Planificación, Presupuesto y Gestión - MP es el órgano central normativo.»

Según Fernandes (2002:3), el Comprasnet «se creó en 1997, para publicar electrónicamente los avisos y los pliegos de licitación (de todas las modalidades) y las actas de los contratos firmados por la Administración Pública Federal. Más tarde, cuando el Decreto $\mathrm{n}^{\circ} 3.697$, de diciembre de 2000, estableció la subasta a la baja electrónica, el Comprasnet pasó a ser utilizado para las adquisiciones en tiempo real, trabajando con este nuevo tipo de licitación. En octubre de 2001, el Comprasnet sufrió una nueva transformación, convirtiéndose en un efectivo portal de compras. La gran innovación, que dio al Comprasnet el estatus de paradigma internacional en el campo de las compras electrónicas gubernamentales, fue la introducción de un modelo de negocios en el que el gobierno federal comparte con socios privados la gestión del portal».

\section{Figura 1. Portal de compras del gobierno federal brasileño en internet}

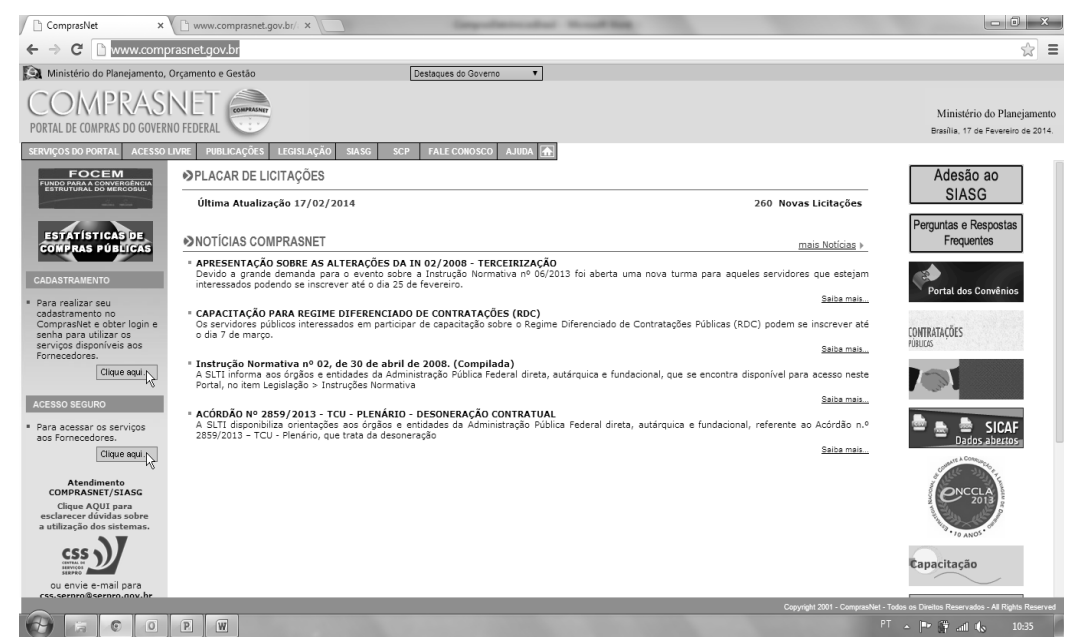

Fuente: http://www.comprasnet.gov.br/

5 Para una visión más amplia de lo que es, como evolucionó y cómo funciona el Comprasnet, ver Brasil (2002). 
La subasta a la baja electrónica se consolidó como procedimiento administrativo en el gobierno federal, aumentando año a año por esta vía el número de compras y contrataciones. De hecho se trata del procedimiento más utilizado (si exceptuamos aquellos supuestos para los que se dispensa de licitación), habiéndose estabilizado su número en unas 34.000 compras públicas anuales, tal y como como muestra la Tabla 1 .

\section{Tabla 1. Cantidad de compras públicas del gobierno federal de Brasil, según el tipo de proceso (2008-2013)}

\begin{tabular}{|l|c|c|c|c|c|c|}
\hline $\begin{array}{l}\text { Modalidad (según } \\
\begin{array}{l}\text { leyes 8.666/93 } \\
\text { y 10.520/02) }\end{array}\end{array}$ & $\mathbf{2 0 0 8}$ & $\mathbf{2 0 0 9}$ & $\mathbf{2 0 1 0}$ & $\mathbf{2 0 1 1}$ & $\mathbf{2 0 1 2}$ & $\begin{array}{c}\text { Hasta } \\
\text { oct. 2013 }\end{array}$ \\
\cline { 2 - 7 } & 771 & 1.319 & 934 & 1.086 & 1.314 & 513 \\
\hline $\begin{array}{l}\text { Concurrencia } \\
\text { nacional }\end{array}$ & 50 & 44 & 105 & 51 & 40 & 8 \\
\hline $\begin{array}{l}\text { Concurrencia } \\
\text { Internacional }\end{array}$ & 8 & 16 & 16 & 11 & 16 & 8 \\
\hline Concurso & 4.957 & 1.499 & 961 & 621 & 452 & 198 \\
\hline Invitación & 246.635 & 235.566 & 211.029 & 186.301 & 169.875 & 128.443 \\
\hline $\begin{array}{l}\text { Dispensa de } \\
\text { Licitación }\end{array}$ & 14.048 & 15.838 & 16.677 & 19.742 & 23.783 & 20.310 \\
\hline $\begin{array}{l}\text { Inexigibilidad de } \\
\text { Licitación }\end{array}$ & 30.865 & 33.700 & 33.696 & 32.357 & 34.747 & 26.192 \\
\hline $\begin{array}{l}\text { Subasta a la baja } \\
\text { Electrónica }\end{array}$ & 1.297 & 1.083 & 657 & 334 & 268 & 127 \\
\hline $\begin{array}{l}\text { Subasta a la baja } \\
\text { Presencial }\end{array}$ & 1.641 & 1.636 & 1.334 & 1.170 & 1.307 & 541 \\
\hline Fijación de Precio & $\mathbf{3 0 0 . 2 7 2}$ & $\mathbf{2 9 0 . 7 0 1}$ & $\mathbf{2 6 5 . 4 0 9}$ & $\mathbf{2 4 1 . 6 7 3}$ & $\mathbf{2 3 1 . 8 0 2}$ & $\mathbf{1 7 6 . 3 4 0}$ \\
\hline Total & & & & & \\
\hline
\end{tabular}

Fuente: Brasil, 2013

Por otra parte, la Tabla 2 nos muestra que el valor alcanzado por las subastas a la baja electrónicas también ha crecido año a año (excepto 2011, por razones que se desconocen). Se puede observar que en los dos últimos períodos analizados (año 2012 y hasta octubre de 2013) los valores de las compras públicas llevadas a cabo por dicho procedimiento son de alrededor del doble de la suma de los valores del resto de procedimientos. 


\section{Tabla 2. Valor de las compras públicas del gobierno federal de Brasil, según el tipo de proceso (2008-2013)}

\begin{tabular}{|l|c|c|c|c|c|c|}
\hline \multirow{2}{*}{$\begin{array}{c}\text { Modalidad (según } \\
\text { leyes 8.666/93 y } \\
\mathbf{1 0 . 5 2 0}\end{array}$} & \multicolumn{5}{|c|}{ Valor) } \\
\cline { 2 - 7 } & $\mathbf{2 0 0 8}$ & $\mathbf{2 0 0 9}$ & $\mathbf{2 0 1 0}$ & $\mathbf{2 0 1 1}$ & $\mathbf{2 0 1 2}$ & $\begin{array}{c}\text { Hasta } \\
\text { oct. 2013 }\end{array}$ \\
\hline $\begin{array}{l}\text { Concurrencia } \\
\text { nacional }\end{array}$ & $9.167 .855 .840,53$ & $14.546 .704 .831,43$ & $12.393 .154 .773,52$ & $6.377 .263 .000,50$ & $12.678 .890 .659,73$ & $2.481 .597 .252,59$ \\
\hline $\begin{array}{l}\text { Concurrencia } \\
\text { Internacional }\end{array}$ & $50146.359 .305,34$ & $1.053 .839 .103,41$ & $1.032 .577 .330,98$ & $85.951 .777,18$ & $140.937 .239,77$ & $5.969 .035,83$ \\
\hline Concurso & $372.625,43$ & $1.151 .347,51$ & $2.974 .844,09$ & $901.562,48$ & $2.581 .625,00$ & $293.136,59$ \\
\hline Invitación & $163.181 .777,33$ & $73.198 .638,70$ & $49.330 .037,37$ & $32.885 .611,90$ & $22.409 .868,60$ & $7.911 .596,22$ \\
\hline Dispensa & $15.134 .384 .857,80$ & $8.725 .194 .787,66$ & $11.676 .471 .129,88$ & $7.646 .697 .849,80$ & $13.802 .507 .425,32$ & $6.484 .101 .171,62$ \\
\hline Inexigibilidad & $3.210 .978 .280,03$ & $5.824 .096 .648,18$ & $7.731 .978 .598,76$ & $11.513 .800 .634,23$ & $10.672 .115 .922,26$ & $8.171 .740 .963,04$ \\
\hline $\begin{array}{l}\text { Subasta a la baja } \\
\text { Electrónica }\end{array}$ & $21.277 .510 .721,75$ & $22.026 .596 .987,24$ & $28.656 .588 .714,71$ & $24.646 .715 .336,42$ & $33.639 .555 .907,00$ & $29.608 .299 .718,86$ \\
\hline $\begin{array}{l}\text { Subasta a la baja } \\
\text { Presencial }\end{array}$ & $2.802 .208 .233,56$ & $3.117 .945 .219,90$ & $1.313 .059 .935,34$ & $1.017 .601 .729,99$ & $1.152 .980 .671,26$ & $398.695 .537,30$ \\
\hline Fijación de Precio & $745.677 .499,84$ & $696.300 .483,06$ & $557.320 .756,89$ & $462.949 .601,53$ & $507.064 .775,89$ & $166.219 .979,01$ \\
\hline Total & $\mathbf{5 2 . 6 4 8 . 5 2 9 . 1 4 1 , 6 0}$ & $\mathbf{5 6 . 0 6 5 . 0 2 8 . 0 4 7 , 0 8}$ & $\mathbf{6 3 . 4 1 3 . 4 5 6 . 1 2 1 , 5 4}$ & $\mathbf{5 1 . 7 8 4 . 7 6 7 . 1 0 4 , 0 3}$ & $72.619 .044 .094,83$ & $47.324 .828 .391,06$ \\
\hline
\end{tabular}

Fuente: Brasil, 2013

c) Subasta a la baja electrónica en los niveles subnacionales de la federación

En los Estados y Municipios también avanzó la utilización de la subasta a la baja y de la subasta a la baja electrónica desde el momento en que la ley 10.520/2002 ofreció seguridad jurídica para que eso ocurriese, con la ayuda del Decreto 5.405, de 2005.

Ya en 2002 algunas experiencias de subasta a la baja electrónica se consolidaron en el nivel de los Estados, como revela Fernandes (2002). Entre ellos los pioneros fueron São Paulo (con la BEC - Bolsa Electrónica de Compras, http://www.bec.sp.gov.br/ Publico/Aspx/Home.aspx), una de las más completas y sofisticadas herramientas en Brasil de public e-Procurement, lanzada antes del Comprasnet del gobierno federal; Bahia (Comprasnet.ba, http://www.Comprasnet.ba.gov.br/); Tocantins (Compr@s.TO, http://www.compras.to.gov.br/scripts/nwwcgi.exe/compras2/home); Rio Grande do Sul (CELIC - Central de Licitaciones, http://www.celic.rs.gov.br/); Mato Grosso do Sul (Central de Compras, http://www.centraldecompras.ms.gov.br/). 
Desde la aprobación de la ley 10.520, de 2002, todos los municipios están legalmente amparados para adoptar la subasta a la baja y la subasta a la baja electrónica. Mientras tanto, como reveló la investigación realizada por la Confederación Nacional de Municipios (2010), la adhesión va aumentando lentamente. De los 2.780 municipios investigados por la CNM (de un total de 5.565 existentes en el país), 2.270 (82\%) utilizaban la subasta a la baja, pero solamente $1.890(68 \%)$ lo utilizaban en la modalidad electrónica, por falta de infraestructura tecnológica y/o por desconfianza al cambio. 998 municipios afirmaron no tener interés en usar la subasta a la baja electrónica. Desde 2010, año de la investigación, es probable que se hayan producido avances, entre otras razones porque existe presión para que esto ocurra (por parte de grupos que combaten la corrupción o que proponen la modernización y también de proveedores deseosos de mayor transparencia y agilidad). También están disponibles diversas plataformas tecnológicas para la implantación del sistema, como las ofrecidas por el Banco do Brasil (LicitaçõesBB, http://www.bb.com.br/portalbb/page3,111,4241,13,0, 1,3.bb), la Caja Económica Federal, Banrisul, CNM (CidadeCompras, http://www. cidadecompras.com.br/1/) y varias empresas que abastecen de soluciones tecnológicas a los gobiernos municipales.

El proceso de innovación de las compras públicas en los municipios brasileños se enfrenta, de hecho, a muchas dificultades, siendo necesarias voluntad política y liderazgo para implementarlo, como señala un estudio reciente del caso de la ciudad de São Carlos, en el Estado de São Paulo (Blanco García, 2010).

\section{d) Resultados obtenidos por la subasta a la baja electrónica}

En el gobierno federal, «En 2013, la subasta a la baja electrónica supuso el 63\% de las compras del gobierno, con un gasto de 29,6 billones de reales, siendo utilizada en 26,2 mil procedimientos (15\%). Si comparamos solamente las modalidades de licitación, esa forma de contratación fue responsable del $91 \%$ de los gastos en adquisiciones, suponiendo un ahorro para las arcas públicas del orden de 6,9 billones de reales (19\%). En relación al número de concursos de licitación, la subasta a la baja electrónica supuso el 95\%» (Brasil, 2013:2).

Esta modalidad de compra se concentra en algunos tipos de bienes y servicios. Según el SLTI/MPOG, refiriéndose a 2013, «Los bienes y servicios más adquiridos por subasta a la baja electrónica pertenecen, respectivamente, a los grupos de equipamientos y artículos para uso médico, dental y veterinario ( 2,8 billones de reales) y servicios de ingeniería (1,6 billones de reales) (Brasil, 2013: 3). La tabla 3 indica los valores para todos los tipos de bienes y servicios adquiridos por medio de subasta a la baja electrónica en 2013 (hasta octubre). 
Tabla 3. Valor de las compras de los grupos de materiales más adquiridos por el gobierno federal de Brasil, mediante subasta a la baja electrónica (enero-octubre 2013)

\begin{tabular}{|l|c|}
\hline \multicolumn{1}{|c|}{$\begin{array}{c}\text { Grupos de Materiales } \\
\text { (adquiridos por subasta a la baja electrónica) }\end{array}$} & $\begin{array}{c}\text { Valor de Compra } \\
\text { (reales brasileños) }\end{array}$ \\
\hline $\begin{array}{l}\text { Equipamientos y artículos para uso médico, } \\
\text { dental y veterinario }\end{array}$ & $2.846 .798 .273,15$ \\
\hline $\begin{array}{l}\text { Equipamiento para procesamiento automático } \\
\text { de datos (incluyendo firmware, software, acce- } \\
\text { sorios y equipamiento de soporte }\end{array}$ & $2.005 .370 .919,45$ \\
\hline Vehículos & $1.683 .797 .713,87$ \\
\hline Mobiliario & $1.444 .692 .439,41$ \\
\hline Subsistencia & $1.328 .351 .138,44$ \\
\hline Materiales de construcción & $687.740 .818,08$ \\
\hline Instrumentos y equipamientos de laboratorio & $669.618 .674,93$ \\
\hline $\begin{array}{l}\text { Vestuario, equipamientos individuales e insig- } \\
\text { nias }\end{array}$ & $629.045 .883,35$ \\
\hline Material de escritorio y material de oficina & $\mathbf{1 8 . 7 0 9 . 3 3 3 . 8 3 1 , 0 9}$ \\
\hline $\begin{array}{l}\text { Equipamiento para combatir incendios, para } \\
\text { rescate y seguridad }\end{array}$ & $448.180 .392,43$ \\
\hline Otros Grupos & $6.448 .632 .906,40$ \\
\hline Total & \\
\hline
\end{tabular}

Fuente: Brasil, 2013

Son también muy importantes los resultados que aporta la subasta a la baja electrónica a las relaciones del gobierno con los proveedores y a la gestión de compras y contrataciones. Las facilidades que tiene un procedimiento menos burocrático y más transparente atraen a una mayor cantidad de interesados en participar de las licitaciones, entre ellos pequeñas y medianas empresas (Brasil, 2013). En cuanto a la innovación en la gestión, es suficiente la navegación por el portal Comprasnet para por lo menos sospechar que fue necesario alterar en profundidad rutinas, procedimientos $y$, sobre todo, la cultura administrativa, a fin de convertirse en el instrumento principal de relación del gobierno con los proveedores. Este portal contiene canales de acceso para uso exclusivo de los compradores públicos, para utilización tanto por los potenciales como por los efectivos proveedores y también facilita información a quien se interese 
por ella (al respecto, ver la solapa «Servicios del Portal» de Comprasnet); facilita acceso libre a los concursos de licitación (solapa «Acceso Libre»), divulga las normas relativas al área (solapa «Legislación») y literatura técnica sobre el tema (solapa «Publicaciones»).

Desde hace poco se van realizando investigaciones, análisis y estudios con el objetivo de evaluar los resultados de la innovación que supone en Brasil, en los diversos niveles de gobierno, la subasta a la baja electrónica. (Santana, 2013; Silveira, Filardi y Freitas, 2012; Mota y Rodrigues, 2010; Figueiredo, 2009; Alves y Dufloth, 2008; Morais y Slomski, 2006; Carneiro y Pereira, 2005). En general, en estos trabajos se señalan avances en la agilización de los procedimientos; la ampliación del número de ofertantes interesados, la reducción de precios; la transparencia y la barrera a la corrupción; y la economía de las compras. Pero surgen dudas sobre la calidad de los productos comprados a precios más bajos (afectando negativamente a la relación coste-beneficio de las compras públicas) y sobre las posibilidades de fraudes en la recepción de propuestas por los sistemas electrónicos. Aparentemente, los estudios mencionados pueden contribuir a la mejora del public e-Procurement en Brasil, que probablemente será incorporado definitivamente en una posible próxima ley de licitaciones y contratos que reúna y sintetice los cambios difusos y dispersos producidos en las dos últimas décadas, en los márgenes de la desfasada ley 8.666/93.

Finalmente, merece la pena reproducir, por la oportunidad y coherencia, la conclusión de Tripadali, Fernandes y Machado (2011:429): «Destacamos que la simple adopción de la compra electrónica no es suficiente para conseguir los máximos resultados en la gestión de los gastos públicos corrientes, pues existen otras oportunidades de mejora si ampliamos el análisis a las demás fases de la cadena de suministro.» Lo mismo debe aplicarse, evidentemente, a todos los gastos y no solo a los corrientes.

\section{España}

\section{a) Antecedentes}

En España el ordenamiento de contratos, tal como hoy lo conocemos, es un fenómeno histórico relativamente reciente. La primera regulación general de contratos públicos apareció en el año 1963, con la aprobación de la Ley de Bases de Contratos del Estado y su texto articulado de 1965. Hasta entonces, la «materia de contratos» no había sido objeto de una regulación específica y diferenciada.

A partir de ese momento la evolución de este sector del ordenamiento jurídicopúblico ha tenido cuatro hitos (Santamaría, 2013): a) el bloque normativo de los años sesenta, constituido por el antes citado texto articulado de 8 de abril de 1965 y sus dos sucesivos Reglamentos, de 1967 (Decreto 3354/1967, de 28 de diciembre) y de 1975 (Decreto 3410/1975, de 25 de noviembre); b) el bloque de los años noventa, motivado por la imperativa incorporación de la normativa comunitaria, e integrado por la Ley 13/1995, de 18 de mayo, de Contratos de las Administraciones Públicas y su Reglamento parcial, aprobado por Real Decreto 390/1996, de 1 de marzo; c) el bloque de comienzo del milenio, constituido por el Texto Refundido de la Ley de Contratos de 
las Administraciones Públicas (Real Decreto Legislativo 2/2000, de 16 de junio) y su Reglamento (esta vez general) aprobado por Real Decreto 1098/2001, de 12 de octubre; uno y otro texto, impulsados por la profunda reforma que la Ley de 1995 sufrió en 1999, entre otras modificaciones; y d) el bloque hoy vigente, iniciado con la Ley 30/2007, de 30 de octubre, de Contratos del Sector Público (LCSP), la refundición hecha por el Real Decreto Legislativo 3/2011, de 14 de noviembre (RDLCSP) y la nueva Directiva 2014/24/UE del Parlamento Europeo y del Consejo ${ }^{6}$.

En cuanto a la transformación hacia una nueva administración electrónica, esta empezó a operarse a nivel de Derecho interno en la primera redacción de la Ley 30/1992, de 26 de noviembre, de Régimen Jurídico de las Administraciones Públicas y del Procedimiento Administrativo Común, en la que se hablaba de la incorporación de medios técnicos y de la informatización de registros y archivos, aunque todo ello enfocado desde un punto de vista facultativo y no imperativo para las administraciones. Sin embargo, no será hasta la Ley 11/2007, de 22 de junio, de acceso electrónico de los ciudadanos a los Servicios Públicos, cuando se dé un salto cualitativo en la configuración normativa del uso de las TIC en el ámbito administrativo, pasando del «podrán» al «deberán»y, por tanto, creando obligaciones para las administraciones a favor de los ciudadanos. Tras esta ley la implantación legal de las nuevas tecnologías se consolidará y extenderá a todos los sectores de la actividad administrativa, entre ellos el ámbito contractual público.

En el proceso de impulso de la contratación pública electrónica no podemos olvidar el papel activo y protagonista que ha venido asumiendo la Unión Europea. Las Directivas de $2004^{7}$, aunque supusieron un paso importante para la contratación electrónica no tuvieron los resultados esperados debido a que no se estableció la obligación para los Estados miembros de implementar necesariamente soluciones de e-Contratación. Ante esta situación, la Comisión Europea publicó en 2010 el Libro Verde sobre la generalización del recurso a la contratación pública electrónica en la Unión Europea, SEC (2010) 1214. Asimismo, en 2011 editó el Libro Verde sobre la modernización de la política de contratación pública de la Unión Europea, con el que puso en marcha una amplia consulta pública sobre los cambios legislativos que podrían introducirse para tratar de facilitar y flexibilizar la adjudicación de los contratos y hacer posible una mejor utilización de los contratos públicos en apoyo de otras políticas.

${ }^{6}$ El cambio normativo ha experimentado un ritmo crecientemente acelerado, acortándose cada vez más las distancias temporales entre cada uno de los bloques: entre el bloque de los sesenta y de los noventa transcurrieron treinta años, que se redujeron a solo seis entre el bloque de los noventa y el del milenio; el mismo número de años que separan el Reglamento de 2001 y la Ley de Contratos del Sector Público de 2007. Y entre el cuarto y último bloque y el que se avecina, con las nuevas Directivas comunitarias en curso de aprobación, transcurrirán solo, posiblemente, tres años (Santamaría, 2013).

7 Directiva 2004/18/CE del Parlamento Europeo y del Consejo, de 31 de marzo de 2004, sobre coordinación de los procedimientos de adjudicación de los contratos públicos de obras, de suministro y de servicios y Directiva 2004/17/CE del Parlamento Europeo y del Consejo, de 31 de marzo de 2004, sobre la coordinación de los procedimientos de adjudicación de contratos en los sectores del agua, de la energía, de los transportes y de los servicios postales. 
Así, tras un proceso de tramitación de más de dos años, finalmente se aprueba una nueva directiva de contratación pública - la Directiva 2014/24/UE del Parlamento Europeo y del Consejo, de 26 de febrero de 2014, sobre contratación pública- que viene a sustituir a la Directiva 2004/18/CE. Esta nueva norma reconoce que los medios de información y comunicación electrónicos sirven para simplificar la publicación de los contratos, aumentando la eficiencia y la transparencia de los procedimientos de contratación, razón por la cual «Deben convertirse en el método estándar de comunicación e intercambio de información en los procedimientos de contratación, ya que hacen aumentar considerablemente las posibilidades de los operadores económicos de participar en dichos procedimientos en todo el mercado interior» (Considerando 52). A su vez, se adopta una comunicación totalmente electrónica, en particular por lo que respecta a la presentación de ofertas o solicitudes de participación, en todos los procedimientos de licitación, con un período de transición de 30 meses $^{8}$.

En relación con los procedimientos de contratación existentes en España, tradicionalmente existían el procedimiento abierto, el negociado y el restringido ${ }^{9}$, que se combinaban con dos formas de contratación (aplicables únicamente al procedimiento abierto y al negociado) que eran la subasta (la adjudicación recaía en quien ofrecía el precio más bajo) y el concurso (la adjudicación se realizaba teniendo en cuenta los criterios establecidos en los pliegos, sin atender exclusivamente al precio). Con la Ley 30/2007 se incorpora a nuestra legislación la terminología comunitaria, a fin de — tal y como señala su Exposición de Motivos - «facilitar, desde el plano semántico, la interoperabilidad con los sistemas europeos de contratación». Se abandonan los anteriores términos de «concurso» y «subasta», de manera que las hasta el momento «formas de contratación» se subsumen en la expresión «oferta económicamente más ventajosa», cuya terminología refuerza la idea de la eficiencia en la contratación, y que remite a los criterios que el órgano de contratación debe tomar en cuenta para valorar las ofertas de los licitadores en los diferentes procedimientos abiertos, restringidos o negociados, bien utilizando un único criterio de adjudicación (el precio, como en la antigua «subasta») o bien varios criterios (como en el anterior «concurso»). Todo ello sin perjuicio de la existencia del contrato menor, que es un procedimiento simplificado a utilizar en contratos de cuantía reducida, exigiéndose únicamente la aprobación del gasto e incorporación de la factura al expediente (en los contratos de obras, además, se aportará el correspondiente presupuesto, y cuando así sea necesario, la apro-

${ }^{8}$ Sin embargo, la propia directiva contiene una limitación, al indicar que la misma «no debe obligar a los poderes adjudicadores a tratar electrónicamente las ofertas, como tampoco debe exigir la evaluación electrónica ni el tratamiento automatizado», y que «ningún elemento del procedimiento de contratación pública tras la adjudicación del contrato debe estar sujeto a la obligación de utilizar medios electrónicos de comunicación, como tampoco debe estarlo la comunicación interna en el marco el poder adjudicador» (Considerando 52).

${ }^{9}$ En el procedimiento abierto, todo empresario interesado puede presentar una proposición; en el procedimiento restringido solamente aquellos empresarios expresamente seleccionados por la administración, previa solicitud de los mismos, pueden presentar proposición; y en el procedimiento negociado el contrato es adjudicado al empresario justificadamente elegido por la administración, previa consulta y negociación de los términos del contrato con uno o varios de ellos. 
bación del proyecto), no teniendo una duración superior a un año ni siendo objeto de prórroga ${ }^{10}$.

Por otra parte, el actual TRLCSP, siguiendo el contenido de la LCSP, recoge los procedimientos de contratación enteramente electrónicos regulados por la normativa comunitaria (sistema dinámico de adquisición y subasta electrónica). A su vez, en su disposición adicional decimosexta, contempla diversas cuestiones de índole general relacionadas con el uso de medios electrónicos, informáticos y telemáticos. Asimismo, se formula una prescripción que directamente vincula tecnología con transparencia: «En cumplimiento del principio de transparencia en la contratación y de eficacia y eficiencia de la actuación administrativa, se fomentará y preferirá el empleo de medios electrónicos, informáticos y telemáticos en los procedimientos contemplados en esta Ley por parte de los licitadores o los candidatos» (disposición adicional decimosexta. Apdo. 3 TRLCSP).

\section{b) Los datos de la e-Contratación en España}

A diferencia de lo que sucede con Brasil, los datos estadísticos ofrecidos por los poderes públicos españoles son casi inexistentes. Y ello a pesar de que sí se han previsto los instrumentos necesarios al respecto, como sucede con el Registro de Contratos del Sector Público. De hecho, dicha institución, dependiente del Ministerio de Hacienda y Administraciones Públicas, a tenor de lo dispuesto por el art. 333 del TRLCSP, se constituye con vocación de convertirse en el sistema oficial central de información sobre la contratación pública en nuestro país ${ }^{11}$.

Los órganos de contratación deben comunicar al Registro de Contratos del Sector Público, para su inscripción, los datos básicos de los contratos adjudicados. También, las modificaciones, prórrogas, variaciones en los plazos o precio, el importe final o la extinción de los mismos. Sin embargo, pese a lo señalado - que recoge la buena voluntad del legislador - la realidad es otra, pues, hoy por hoy, no se explota el potencial que el propio Registro de Contratos posee. Así por ejemplo, en el momento actual (julio de 2014) la última información publicada en la página web de la entidad es un escueto «Resumen general de contratos 2012» con datos «Provisionales a fecha 26/12/2013», sin que exista la más mínima referencia sobre cuál ha sido la repercusión de la contratación pública electrónica durante estos últimos años.

10 Arts. 111 y 138.3 TRLCSP.

11 A tal fin se configura como «soporte para el conocimiento, análisis e investigación de la contratación pública, para la estadística en materia de contratos públicos, para el cumplimiento de las obligaciones internacionales de España en materia de información sobre la contratación pública, para las comunicaciones de los datos sobre contratos a otros órganos de la Administración que estén legalmente previstas $\mathrm{y}$, en general, para la difusión pública de dicha información, de conformidad con el principio de transparencia». Asimismo, constituirá «el instrumento de los poderes públicos para la revisión y mejora continuas de los procedimientos y prácticas de la contratación pública, el análisis de la calidad, fiabilidad y eficiencia de sus proveedores, y la supervisión de la competencia y transparencia en los mercados públicos» (art. 333. 2. TRLCAP). 
Tampoco la Plataforma de Contratación del Sector Público, como plataforma electrónica para dar publicidad a través de internet a las convocatorias de licitaciones y sus resultados, así como de aquella información que se considera relevante relativa a los contratos que celebren (art. 334 TRLCSP), ofrece datos al respecto.

\section{Figura 2. Plataforma de Contratación del Sector Público en España}

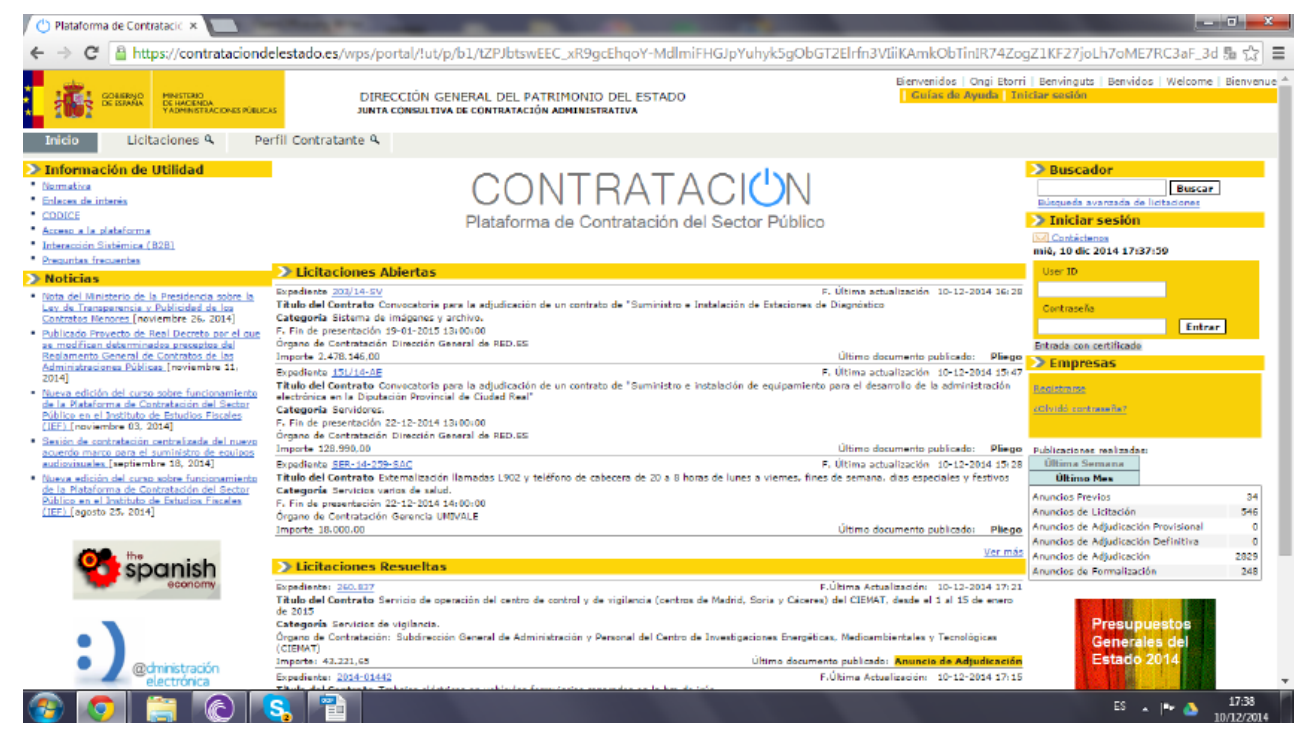

Fuente: https://contrataciondelestado.es/

Por lo tanto, independientemente de la mayor o menor incidencia de la e-Contratación en España, existe un problema de fondo que es el del tratamiento de la información sobre contratación pública electrónica. Lo que supone una clara limitación al principio de transparencia, entrando en contravención con las actuales iniciativas normativas en favor de la misma ${ }^{12}$. Habiéndose de tomar en cuenta que, al parecer, hoy en día se dispone de los instrumentos legales y técnicos para poder implementarla convenientemente. De hecho, en el estudio bianual realizado por Naciones Unidas sobre el

12 El propio Texto Refundido de la Ley de Contratos del Sector Público (Real Decreto Legislativo 3/2011), ya en su artículo 1 incluye entre los principios que han de regir la contratación el de publicidad y transparencia de los procedimientos, además del de libre acceso a las licitaciones y no discriminación e igualdad de trato entre los candidatos. Asimismo, de una forma relativamente reciente, aunque no sin cierto retraso respecto de los países de su entorno, España se ha incorporado al grupo de Estados que disponen de una norma específica que regule la transparencia en el ámbito público, en este caso la Ley 19/2013, de 9 de diciembre de transparencia, acceso a la información pública y buen gobierno. 
desarrollo de la administración electrónica ${ }^{13}$, de los 193 países analizados, en 2014 España se sitúa en el puesto 12 en el grado de implantación de la Administración electrónica (mejorando once posiciones respecto de las puntuaciones obtenidas en el anterior estudio de 2012), mientras que Brasil se halla en el puesto 57 (la mejora es de dos posiciones).

Tabla 4. Grado de implementación de la administración electrónica en España y Brasil (2004-2014)

\begin{tabular}{|c|c|c|c|c|c|c|}
\hline & $\mathbf{2 0 0 4}$ & $\mathbf{2 0 0 5}$ & $\mathbf{2 0 0 8}$ & $\mathbf{2 0 1 0}$ & $\mathbf{2 0 1 2}$ & $\mathbf{2 0 1 4}$ \\
\hline España & 34 & 39 & 20 & 9 & 23 & 12 \\
\hline Brasil & 35 & 33 & 45 & 61 & 59 & 57 \\
\hline
\end{tabular}

Fuente: UNPCAS, 2014

Asimismo, siguiendo dicho estudio, en relación con el uso de servicios electrónicos, España se halla en el puesto 19 (doce posiciones de mejora respecto a 2012) y Brasil en el puesto 24 (su mejora es de siete posiciones).

Tabla 5. Grado de uso de los servicios públicos electrónicos en España y Brasil (2004-2014)

\begin{tabular}{|c|c|c|c|c|c|c|}
\hline & $\mathbf{2 0 0 4}$ & $\mathbf{2 0 0 5}$ & $\mathbf{2 0 0 8}$ & $\mathbf{2 0 1 0}$ & $\mathbf{2 0 1 2}$ & $\mathbf{2 0 1 4}$ \\
\hline España & 97 & 73 & 34 & 3 & 31 & 19 \\
\hline Brasil & 35 & 18 & 23 & 42 & 31 & 24 \\
\hline
\end{tabular}

Fuente: UNPCAS, 2014

Por otra parte, no podemos olvidar que, como veíamos anteriormente, la contratación pública en España se desarrolla dentro de un contexto regional amplio y variado, que es el conformado por los países de la Unión Europea. Así pues, la falta de obliga-

13 En dicho estudio el gobierno electrónico se conceptúa como el uso de las tecnologías de la información y la comunicación (TIC) para una mayor eficacia y prestación efectiva de los servicios gubernamentales a ciudadanos y empresas. Se trata, pues, de la aplicación de las TIC para el logro de los fines públicos por medios digitales. 
toriedad de la contratación pública electrónica para el ámbito de la Unión Europea, derivada de la anterior directiva de contratos, ha supuesto una utilización desigual de esta figura en dicho ámbito territorial. De hecho, mientras en Portugal la generalidad de la contratación administrativa se lleva a cabo de forma electrónica desde el año 2009, en España esta obligación no ha existido, lo que redunda en una incidencia limitada de la misma.

De esta forma, los datos de España respecto del conjunto de la Unión ofrecen un panorama ciertamente limitado:

\section{Tabla 6. Porcentaje de empresas que han usado los sistemas públicos de e-Contratación en Europa (2012)}

\begin{tabular}{|l|c|c|c|c|c|c|}
\hline & UE & España & Francia & Alemania & Portugal & Italia \\
\hline $\begin{array}{l}\text { Empresas que han usado internet para } \\
\text { acceder a la documentación y las especi- } \\
\text { ficaciones técnicas de las licitaciones en } \\
\text { sistemas públicos de e-Contratación }\end{array}$ & 19 & $\mathbf{3 1}$ & 3 & 34 & 73 & 97 \\
\hline $\begin{array}{l}\text { Empresas que han ofrecido bienes o ser- } \\
\text { vicios mediante sistemas públicos de e- } \\
\text { Contratación }\end{array}$ & 24 & $\mathbf{3 1}$ & 42 & 23 & 18 & 35 \\
\hline
\end{tabular}

Fuente: EUROSTAT, 2013

Siendo que la contratación electrónica tiende a reducir la discrecionalidad de la administración a la hora de valoración de las ofertas, lo lógico es que con la futura implantación obligatoria de la misma, disminuya el número de contratos que contienen mayores componentes de subjetividad (como han venido siendo los tradicionales «concursos» $\mathrm{o}$ «procedimientos negociados») y se potencien otras formas de adjudicación como son aquellas que tienen en cuenta únicamente criterios de carácter objetivo, fundamentalmente el precio (como ocurre con la anterior «subasta», hoy «procedimiento con un único criterio de adjudicación» que es necesariamente el precio, y también con los procedimientos enteramente electrónicos como la subasta electrónica ${ }^{14} \mathrm{y}$ los sistemas dinámicos de adquisición).

De hecho, el propio proceso evolutivo del número total de contratos por forma de adjudicación (Tabla 7) nos muestra que la tradicional subasta si bien actualmente se mantiene en una posición de segundo orden respecto del resto de procedimientos que

${ }^{14}$ Cabe destacar que incluso la Comisión Nacional del Mercado de Valores, en su «Guía sobre Contratación Pública y Competencia» (2011:43) ya advertía de la necesidad de acudir a las subastas electrónicas ya que, por su transparencia, pueden facilitar la detección de acuerdos de colusión entre empresas y el conocimiento de precios y comportamientos de competidores. 
contienen mayor carga de subjetividad en su conjunto (concurso y procedimiento negociado), la misma ha ido ganando posiciones a lo largo del período 2009-2012, con un salto excepcional de 2011 a 2012 (más de un 400\% de aumento).

Tabla 7. Número total de contratos en España por modalidad de adjudicación (2006-2012)

\begin{tabular}{|l|c|c|c|c|c|c|c|}
\hline & $\mathbf{2 0 0 6}$ & $\mathbf{2 0 0 7}$ & $\mathbf{2 0 0 8}$ & $\mathbf{2 0 0 9}$ & $\mathbf{2 0 1 0}$ & $\mathbf{2 0 1 1}$ & $\mathbf{2 0 1 2}$ \\
\hline CONCURSO & 68.829 & 67.883 & 59.264 & 54.381 & 55.160 & 56.052 & 56.315 \\
\hline NEGOCIADO & 60.542 & 60.637 & 54.722 & 53.877 & 66.492 & 58.315 & 28.720 \\
\hline SUBASTA & 5.396 & 4.778 & 3.237 & 5.286 & 6.218 & 7.498 & 31.610 \\
\hline TOTAL & $\mathbf{1 3 4 . 7 6 7}$ & $\mathbf{1 3 3 . 2 9 8}$ & $\mathbf{1 1 7 . 2 2 3}$ & $\mathbf{1 1 3 . 5 4 4}$ & $\mathbf{1 2 7 . 8 7 0}$ & $\mathbf{1 2 1 . 8 6 5}$ & $\mathbf{1 1 6 . 6 4 5}$ \\
\hline
\end{tabular}

Fuente: Registro de Contratos del Sector Público, 2014

Aunque también debemos reconocer que si esta es la situación respecto del número de contratos, no sucede lo mismo respecto de las cuantías totales. Como indica la tabla 8 , tanto el concurso como el procedimiento negociado superan ampliamente las cantidades económicas adjudicadas mediante subasta:

Tabla 8. Importe total de contratos en España, en euros, por modalidad de adjudicación (2006-2012)

\begin{tabular}{|l|c|c|c|c|c|c|c|}
\hline & $\mathbf{2 0 0 6}$ & $\mathbf{2 0 0 7}$ & $\mathbf{2 0 0 8}$ & $\mathbf{2 0 0 9}$ & $\mathbf{2 0 1 0}$ & $\mathbf{2 0 1 1}$ & $\mathbf{2 0 1 2}$ \\
\hline CONCURSO & 29.343 .461 .810 & 36.929 .570 .635 & 34.351 .865 .333 & 35.517 .835 .321 & 34.879 .508 .891 & 21.828 .779 .969 & 18.481 .412 .433 \\
\hline NEGOCIADO & 13.589 .880 .651 & 12.196 .423 .775 & 13.366 .522 .027 & 13.051 .747 .638 & 12.723 .354 .287 & 8.054 .443 .540 & 3.357 .782 .282 \\
\hline SUBASTA & 2.408 .800 .430 & 2.366 .489 .377 & 2.251 .307 .755 & 2.654 .636 .501 & 2.179 .093 .324 & 2.427 .281 .535 & 2.206 .372 .475 \\
\hline TOTAL & $\mathbf{4 5 . 3 4 2 . 1 4 2 . 8 9 1}$ & $\mathbf{5 1 . 4 9 2 . 4 8 3 . 7 8 7}$ & $\mathbf{4 9 . 9 6 9 . 6 9 5 . 1 1 5}$ & $\mathbf{5 1 . 2 2 4 . 2 1 9 . 5 0 5}$ & $\mathbf{4 9 . 7 8 1 . 9 5 6 . 5 0 2}$ & $\mathbf{3 2 . 3 1 0 . 5 0 5 . 0 4 4}$ & $\mathbf{2 4 . 0 4 5 . 5 6 7 . 1 9 0}$ \\
\hline
\end{tabular}

Fuente: Registro de Contratos del Sector Público, 2014. 
Finalmente, si nos remitimos a los tres niveles de gobierno existentes en España estatal, autonómico y local- debemos tomar en cuenta que las propuestas más desarrolladas de contratación pública electrónica tienen su origen en el nivel inferior al estata $l^{15}$. De lo que se deduce que no ha existido una política clara común y liderada por el gobierno central de apuesta por el modelo de public e-Procurement, sino que la implementación del mismo viene conformada de forma fragmentada, mediante meritorias iniciativas de carácter aislado.

\section{Conclusiones}

Tanto Brasil como España se hallan en un entorno global con unos problemas que - aunque no exentos de peculiaridades en cada supuesto - comparten, en cierto modo, elementos o problemáticas comunes: corrupción, desafección, crisis económica, etc. Sin embargo, las propuestas de innovación vinculadas a la contratación pública electrónica, que se vienen desarrollando en uno u otro caso, adoptan unas características ciertamente diferenciadas.

De un lado, podemos apreciar que el proceso evolutivo de ambos modelos ha discurrido por caminos separados, con influencias también diferentes: en Brasil, fruto de un desarrollo «natural» de sus propios modalidades de contratación; en España, «a golpe» de normativa europea (o incluso, podemos indicar, «a pesar de» la normativa europea, dado que, hasta la aprobación de la reciente directiva de contratación pública de 2014, no se había previsto la obligatoriedad de la instauración del modelo de la e-Contratación).

Por otra parte, también es remarcable que, pese al menor grado de implementación de la administración electrónica y del uso de los servicios públicos electrónicos en el caso de Brasil, lo cierto es que los datos arrojan un aparente mayor desarrollo de la contratación pública electrónica. Resultando, hasta cierto punto preocupante, el hecho de que, en el modelo español, los datos oficiales apenas existen, por lo que resulta prácticamente imposible realizar una estimación completa de dicho grado de penetración. Y, aunque sí se han previsto los instrumentos legales necesarios para llevar a cabo dicha tarea - el Registro de Contratos del Sector Público, fundamentalmente-, los mismos no ofrecen los resultados prometidos.

En cualquier caso, sin un liderazgo sólido, una apuesta decidida por las políticas de innovación (abierta a la participación de la totalidad de los agentes implicados) y una mejora de las condiciones que favorezcan la confianza en el sistema (lo que requiere la transparencia de datos y de procesos) difícilmente se podrá ir más allá de la actual situación en materia de contratación pública electrónica que viven uno y otro país.

$15 \mathrm{Al}$ respecto, merecen especial atención iniciativas como el Modelo Público de Contratación Electrónica del Gobierno Vasco, el Sistema de Contratación Electrónica de la Universidad de Almería y el Sistema de Contratación Pública Electrónica del Ayuntamiento de Gijón (Romeu et al., 2014). Asimismo, en el nivel autonómico, las 17 Comunidades Autónomas tienen accesible el perfil de contratante y los servicios on line de contratación pública, pero tan solo 6 de ellas cuentan con plataforma propia de contratación electrónica (Cataluña, Comunidad Valenciana, Extremadura, La Rioja, y País Vasco). 


\section{Bibliografía}

Ades, A. y Di Tella, R. (1997). «The New Economics of Corruption: A Survey and Some New Results», en Heywood, P. Political Corruption, Blackwell Pub., Oxford.

Agranoff, R. y McGuire, M. (2003). Collaborative Public Management. Georgetown University Press. Washington DC

Aguilar, L.F. y Bustelo, M. (2010). «Gobernanza y evaluación: una relación potencialmente fructífera», en Gestión y Análisis de Políticas Públicas, no 4, pp. 23-51.

Alaweti, M.F., Nurdiana, A. y Faryadi, Q. (2013). «The transparency of public bidding and contracting using e-Procurement in Malaysia», en SMPPS. International Conference on Social Science Research, Penang, Malasia, 4-5 junio 2013.

Alvarez, J.M., Labra, J.E., Cifuentes, F., Alor-Hernandez, G., Sanchez, C. y Guzman J.A. (2011). «Towards a pan-European e-Procurement platform to aggregate, publish and search public procurement notices powered by linked open data: the moldeas approach», en International Journal of Software Engineering and Knowledge Engineering.

Alves, M.V.C. y Dufloth, S.C. (2008). «Portais Eletrônicos de Compras da Administração Pública: Contribuição para Avaliação da Governança Eletrônica no Brasil», en Revista Gestão e Tecnologia, enero-junio, $\mathrm{n}^{\mathrm{o}} 8$, vol. 1, pp. 1-8.

Anderson, CH. y Tverdova, Y. (2003). «Corruption, political allegiances, and attitudes toward government in contemporary democracies», en American Journal of Political Science, $\mathrm{n}^{\mathrm{o}}$ 47, pp. 91-109.

Blanco García, A. del et al. (2010). Eficiencia y transparência em las compras públicas. Análisis de dos casos (São Carlos y Las Palmas de Gran Canaria). Instituto de Estudios Fiscales, Madrid.

Bowler, S. y Karp, J.A. (2004). «Politicians, scandals, and trust in government», en Political Behavior, $\mathrm{n}^{\circ}$ 26, vol. 3, pp. 271-287.

Brasil. (2002). Ministério do Planejamento, Orçamento e Gestão (MPOG). Secretaria de Logística e Tecnologia da Informação (SLTI). SIASG/ Comprasnet: A Tecnologia da Informação na Gestão das Compras Governamentais na Administração Pública Federal Brasileira.

Brasil. (2013). Ministério do Planejamento, Orçamento e Gestão (MPOG). Secretaria de Logística e Tecnologia da Informação (SLTI). Informações Gerenciais de Contratações e Compras Públicas - janeiro a outubro de 2013.

Brugué, Q, Blanco, I. y Boada, J. (2013). «Entornos y motores de la innovación en la Administración Pública», en IV Congreso Internacional en Gobierno, Administración y Políticas Públicas GIGAPP-IUIOG. Madrid.

Caño Gómez, M. J. (2013). «La contratación pública electrónica como palanca productiva en la creciente digitalización de la economía», en IV Congreso Internacional en Gobierno, Administración y Politicas Públicas GIGAPP-IUIOG. Madrid.

Carneiro, R. y Pereira, A.V. (2005). «Os Impactos e os Limites da Implantação do Subasta a la baja como Nova Modalidade de Licitação: uma Abordagem a partir da Experiência do Governo Mineiro», en Encontro da ANPAD, 29, Rio de Janeiro. Anais... Rio de Janeiro: ANPAD.

Confederação Nacional de Municípios (CNM). (2010). «Pesquisa sobre a aplicabilidade do subasta a la baja eletrônico nos municípios brasileiros».

Della Porta, D. y Vanucci, A. (1997). «The 'Perverse Effects' of Political Corruption». En Heywood, P. Political Corruption, Blackwell Pub., Oxford. 
Dreher, A. y Herzfeld, T. (2005). The Economic Costs of Corruption: A Survey and New Evidence.

Elezi, E. y Harizaj, M. (2012). «Efficiency evaluation of the public e-Procurement system in the reduction of curruption: the Albanian case», en Transatlantic Conference on Transparency Research, Utrecht, 7-9 junio 2012.

Eurostat. (2014). Public procurement electronic systems 2013.

Fernandes, A.C.G. (2002). «Compras governamentais eletrônicas no Brasil: Como funcionam os principais sistemas em operação».

Figueiredo, C.A.S. (2009). «Subasta a la baja eletrônico através da BEC/SP - Bolsa Eletrônica de Compras do Estado de São Paulo. Estudo de caso: Rotinas internas para a realização do subasta a la baja eletrônico».

Gardenal, F. (2010). «Public e-Procurement: define, measure and optimize organizational benefits», en 4th International Public Procurement Conference, Seul, Corea del Sur, 26-28 agosto 2010.

González Alonso, A. (2009). «La contratación pública electrónica», en Cuadernos de Derecho Público, no 37 , pp.139-175.

Hedstrom, Peter. (2005). Dissecting the social: on the principles of analytical sociology. Cambridge University Press, Cambridge.

Hodson, G.M. y Jiang, S. (2007). «The economics of corruption and the corruption of economics: an institutionalist perspective», en Journal of Economic Issues, n ${ }^{\circ} 41$, vol. 4, pp. 1043 1061.

Joongi, K. (2006). National Integrity Systems. Transparency International. Country Study Report. Republic of Korea.

Morais, R. de O. y Slomski, V. (2006). «Subasta a la baja Presencial e Subasta a la baja Eletrônico: Instrumentos de Agilidade e Economicidade ao Processo de Licitação Pública», en Encontro de Administração Pública e Governaça, Rio de Janeiro. Anais... Rio de Janeiro: ANPAD.

Moran, F. (2004). «Building a Culture of Innovation in the Public Sector», en Paper Publisher for the Changing the Way Government Works Seminar, Melbourne 5 October

Moreno Molina, J.A. (2011). «Prólogo», en Domínguez Macaya, Jaime (2011). Claves para una contratación pública electrónica eficaz. Madrid: La Ley.

Mota, F.P.B. y Rodrigues Filho, J. (2010). «E-Procurement Público Brasileiro: Nem Só de Lances se Faz um Subasta a la baja», en Encontro de Administração Pública e Governaça, 34, Rio de Janeiro. Anais... Rio de Janeiro: ANPAD.

Neupane, A., Soar, J. y Vaidya, K. (2012a). «Evaluating the anti-corruption capabilities of public e-Procurement in a developing country», en The Electronic Journal on Information Systems in Developing Countries, $\mathrm{n}^{\circ}$ 55, vol. 2, pp. 1-17.

Neupane, A., Soar, J., Vaidya y K. Yong, J. (2012b). «Role of e-procurement technology to reduce corruption in government procurement», en 2012 International Public Procurement Conference, Seattle, Washington, 17-19 agosto 2012.

Osborne, S. y Brown, K. (2005). Managing Change and Innovation in Public Service Organizations. Psychology Press.

Pharr, S.J. y Putnam, R.D. (2000). Disaffected Democracies, Princeton University Press, Princeton, NJ.

Pintos, J. (2014). «Inmediatez y régimen transitorio de la implantación obligatoria de la contratación pública electrónica en las nuevas Directivas sobre contratación pública», en Contratación Administrativa Practica, no 14, pp. 129, pp. 56-65.

Ramírez-Alujas, Á. (2012). «Los nuevos desafíos de la Dirección Pública Profesional: Innovación abierta, gobernanza colaborativa, co-creación y pensamiento de diseño en la gestión», 
en Paper for delivery at the XXII World Congress of Political Science. IPSA-AISP(8-12 July).

Ramírez-Alujas, Á. y Villoria, M. (2012). «Innovaciones de raíz democrática en la Administración: ¿recuperando legitimidad ante la crisis?», en Ekonomiaz, nº 80, pp. 20-45.

Rezende, Flávio da Cunha. (2011). «Razões emergentes para a validade dos estudos de caso na ciência política comparada», en Revista Brasileira de Ciência Politica, nº 6, pp. 297-337.

Romeu, J., Juárez, G. y Pineda, C. (2014a). «La contratación pública electrónica como medio para hacer efectiva la transparencia administrativa», en Revista de Estudios Locales, $\mathrm{n}^{\circ} 168$, pp. 52-74.

Romeu, J., Pineda, C. y Juárez, G. (2014b). «Efectos de la implantación de un sistema público de contratación por medios electrónicos y su incidencia en el panorama español: más allá de un cambio de formato», en Balcells, J., Cerrillo i Martínez, A., Peguera, M., PeñaLópez, I., Pifarré de Moner, M.J., \& Vilasau Solana, M. (coords.), Internet, Derecho y Politica. Una década de transformaciones. Actas del X Congreso Internacional, Internet, Derecho y Política. UOC, Barcelona 3 y 4 de julio de 2014. UOC-Huygens Editorial, Barcelona, pp. 673-690.

Santamaría Pastor, J.A. (2013). «La constante e interminable reforma de la normativa sobre contratación pública», en Cano, T. y Bilbao, E. (coord.). La contratación pública: problemas actuales, Secretaria General de la Comunidad de Madrid.

Santana, P.R. de. (2013). «Sistema Eletrônico de Compras Governamentais: O desempenho do SIASG-Comprasnet e sua influência para o futuro das compras governamentais».

Silveira, F., Filardi, F. y Freitas, A.S. de. (2012). «Subasta a la baja Eletrônico: Uma Análise dos Resultados da Implantação nas Contratações da Administração Pública: O Caso do IFRJ», en Encontro da ANPAD, 36, Rio de Janeiro. Anais... Rio de Janeiro: ANPAD.

Tripadalli, J.P., Fernandes, E. y Machado, W. V. (2011). «Gestão da cadeia de suprimento do setor público: uma alternativa para controle de gastos correntes no Brasil», en Revista de Administração Pública, mar.-abr, no 45, vol. 2, pp. 401-33.

United Nations Public Administration (UNPACS) (2014). UN E-Government Survey.

Vaidya K. (2009). Electronic Procurement: Impact on Procurement Performance. Koln: Lambert Academic Publishing.

Valadares Tavares, L. (2013). «Por qué es necesaria la contratación pública electrónica», en $1^{\circ} s t$ European Conference on e-Public Procurement. Barcelona.

Van Evera, S. (2007). Guide to Methods for Students of Political Science: Cornell University Press, London.

Villoria, M. (2010). «La ética de la evaluación», en Temas para el Debate, no 192, pp. 31-34.

Villoria, M. y Jiménez, F. (2012). «La corrupción en España (2004-2010): datos, percepción y efectos», en REIS, $n^{\circ} 138$, pp. 109-134.

World Bank. (2006). Anti-corruption [online]. ¡Error! Referencia de hipervínculo no válida. [acceso: 30/07/14]

Zapico, E. (2012). «Presentación», en Ekonomiaz, nº 80, pp. 7-19. 